\title{
Spirituality Moderates the Relationship of Psychosocial Stress to Metabolic Risk Factors Among Afro-Caribbean Immigrants in the US Virgin Islands
}

\author{
Eugene S. Tull • Willa M. Doswell • Malcolm A. Cort
}

Received: 24 June 2014 / Revised: 13 August 2014 / Accepted: 29 August 2014 / Published online: 1 October 2014

(C) W. Montague Cobb-NMA Health Institute 2014

\begin{abstract}
Objective Spirituality may contribute to the health advantage of foreign-born blacks compared to United States (US)-born blacks. The objective of this study was to test the hypothesis that spirituality attenuates the association of psychosocial stress to stress-associated metabolic risk factors among foreign-born Caribbean blacks living in a US jurisdiction.

Methods Data on demographic factors, anthropometric measurements (height, weight, and waist), fasting glucose and insulin, lifestyle behaviors (smoking and alcohol use), psychosocial stress, and spirituality were collected from a population-based sample of 319 Afro-Caribbean immigrants, ages 20 and older, who were recruited between 1995 and 2000 in the Virgin Islands of the United States (USVI). Glucose and insulin measurements were used to estimate insulin resistance by the homeostasis model assessment (HOMA-IR) method. Participants were classified into three levels of spirituality, "low," "moderate," and "high," based on the distribution of spirituality scores. Stepwise regression analyses were used to identify the significant predictors of waist circumference and HOMA-IR within each level of spirituality.

Results The predictors of waist circumference and HOMA-IR varied across the levels of spirituality. Psychosocial stress was
\end{abstract}

\section{E. S. Tull $(\bowtie)$}

Inter-American Center for Public Health Improvement, Inc, P.O. Box 3414, Christiansted, St. Croix, VI 00822, USA

e-mail: drmirt@yahoo.com

\section{W. M. Doswell}

Department Health Promotion and Development, School of Nursing, University of Pittsburgh, 440 Victoria Building, 3500 Victoria Street, Pittsburgh, PA 15261-2543, USA

e-mail:wdo100@pitt.edu

\section{A. Cort}

Department of Behavioral Sciences, Athens State University, $300 \mathrm{~N}$

Beaty Street, Athens, AL 35611, USA

e-mail: Malcolm.Cort@athens.edu an independent predictor of waist and HOMA-IR only among participants with a low level of spirituality.

Conclusion Spirituality appears to attenuate the association of psychosocial stress to waist circumference and insulin resistance among Afro-Caribbean immigrants in the USVI.

Keywords Waist circumference $\cdot$ Insulin resistance Spirituality $\cdot$ Psychosocial stress $\cdot$ Afro-Caribbean

\section{Introduction}

Despite being the focus of government policy and research in the United States of America (USA) since the mid-1980s [1], the disparities experienced by black relative to white Americans in the frequency and care for many chronic diseases have been remarkably intractable $[2,3]$. To ascertain the reason(s) for the persistence of these health disparities among black Americans, researchers in the USA have begun to examine the life experience of foreign-born blacks who generally have chronic disease-related morbidity and mortality rates that are lower than those for US-born blacks and, in some instances, comparable to those for whites $[4,5]$. One study utilizing this approach is the National Survey of American Life (NSAL) which includes national probability samples of blacks of English-speaking Caribbean origin, African Americans (descendants of Africans who were enslaved in the USA), and white Americans [6]. A recent report from the NSAL shows that blacks born on the English-speaking Caribbean islands, commonly known as Afro-Caribbean people, have a lower frequency of chronic illnesses than US-born blacks irrespective of whether they are of African American or AfroCaribbean descent [7]. Consequently, research interest has now turned to elucidating factors that contribute to a reduced risk of chronic diseases among Afro-Caribbean immigrants who reside in US jurisdictions. 
Spirituality is one factor that might contribute to a lower chronic disease risk among Afro-Caribbean immigrants compared to African Americans. In the NSAL, African American and Afro-Caribbean participants were found to be similar with respect to their views on the importance of spirituality and the level of self-rated spirituality [8]. However, the two groups differed in that spirituality was associated positively with education, as an indicator of socioeconomic status (SES), among the African American but not the Afro-Caribbean participants [8]. Studies in various ethnic groups have found that a higher level of spirituality is associated with a greater likelihood of using positive coping strategies $[9,10]$ and that positive coping linked to spirituality is associated with a lower level of emotional distress and the stress hormone cortisol [11]. Psychosocial and emotional stress are associated with perturbations in cortisol regulation that are linked to stressassociated risk factors like abdominal obesity and insulin resistance $[12,13]$, which are key risk factors for chronic diseases like type 2 diabetes and cardiovascular disease [14]. Further, it is well established that in the USA, abdominal obesity and insulin resistance are inversely related to SES in various ethnic groups $[15,16]$. Therefore, if the protective health effects of spirituality are distributed across all SES strata in the Afro-Caribbean immigrant population, this may provide an advantage over African Americans for whom higher levels of spirituality tend to be manifested at higher levels of SES where chronic disease risk is lower.

The investigators are not aware of any study that has reported on the association of chronic disease risk factors, such as abdominal obesity and insulin resistance, with spirituality in Afro-Caribbean immigrants living in US jurisdictions. Thus, the aim of the current study was to evaluate the hypothesis that spirituality may help buffer the physiological effects of psychosocial stress among Afro-Caribbean immigrants, such that the association of psychosocial stress with waist circumference and insulin resistance will be strongest at the lowest levels of spirituality and weakest or absent at the highest levels of spirituality. To accomplish this aim, the investigators conducted a study among Afro-Caribbean peoples who reside in the Virgin Islands of the United States (USVI), an insular area of the USA, located in the Caribbean Sea.

The USVI is a cluster of four inhabited islands along with 50 smaller islets and cays that were purchased by the USA from Denmark in 1917 [17]. At the time these islands became possessions of the USA, they had a population of 26,000 persons of whom the vast majority were the descendants of black Africans who had worked at the Danish sugar plantations as slaves [18]. A large wave of Afro-Caribbean migrants, mostly from neighboring islands of the eastern Caribbean (i.e., Antigua, Barbados, St. Kitts-Nevis, Dominica, and St. Lucia), began entering the USVI in the 1960s when the territory underwent development as a destination for tourists from the
USA [18]. Lured by economic opportunities, these AfroCaribbean immigrants helped to triple the population of the USVI between 1960 and 1980 from 32,099 to 96,951 [19]. However, upon their arrival to the USVI, they were viewed as unwelcomed competitors for jobs by the local population and consequently experienced discrimination and stigmatization as "aliens" [18]. Until recent years, the political climate in the USVI may have helped to perpetuate the feeling of being an outsider among many Afro-Caribbean immigrants living in this US territory [18]. Although many of these migrating Afro-Caribbean peoples from the eastern Caribbean stayed in the USVI, others went to the US mainland where they joined with larger numbers of immigrants from bigger Caribbean islands such as Jamaica and Trinidad to comprise the Afro-Caribbean population living in the continental USA.

\section{Methods}

\section{Study Population}

The NSAL dataset, which has been made available to investigators seeking to evaluate new hypotheses, would have been useful for the current assessment but lacks the quantitative data on fasting blood glucose and insulin which are needed for estimating insulin resistance. Therefore, the investigators utilized previously collected data for Afro-Caribbean immigrants ages 20 and older who had participated in a population-based study to identify risk factors for diabetes mellitus and cardiovascular diseases in the USVI [20] and for whom data relating psychosocial stress to waist circumference and insulin resistance have been previously published [21]. In the USVI, AfroCaribbean immigrants are exposed to lifestyle practices that are a facsimile of the US mainland.

The USVI study sample included 406 Afro-Caribbean immigrants for whom data on spirituality, psychosocial stress, waist circumference, and insulin resistance had been ascertained. These individuals represent approximately $77 \%$ of the Afro-Caribbean immigrants who were recruited for the study. The study participants were randomly selected between 1995 and 1999 from households that were randomly chosen from a list of customers (which included approximately $98 \%$ of all households on the island of St. Croix) of the Virgin Islands Water and Power Authority. A consent form approved by the Biomedical Institutional Review Board of the University of Pittsburgh (where the principal investigator was employed) was signed by each person who agreed to participate in the study. Seventy-three of the study participants were found to be diabetic and were excluded from the current assessment because the level of fasting insulin, a key study variable, can be influenced by the treatment of diabetes mellitus. With the exclusion of these individuals, a final 
sample of 333 Afro-Caribbean immigrants was used in analyses in the current study.

\section{Data Collection}

Demographic data were ascertained from participants with an in-home interview. At the time of the interview, participants were scheduled for a visit to the study clinic. At the study clinic, each participant was measured for weight and height, and a venous blood specimen was drawn into a syringe after an overnight fast of 10 to $12 \mathrm{~h}$. Height and weight were measured using standardized techniques [22]. Weight was measured using a balance beam scale with participants standing without shoes in the middle of the platform with head erect and eyes looking straight ahead. With the participant standing without shoes on a level floor, height was measured using a $200-\mathrm{cm}$ aluminum ruler fastened vertically against the wall. The body mass index (BMI), an estimate of overall body size, was calculated for each participant as weight in kilograms $(\mathrm{kg})$ divided by height in meters squared $\left(\mathrm{m}^{2}\right)$ [23].

Sera from the fasting blood samples were measured for serum glucose and insulin. Glucose was analyzed at the Juan F. Louis Hospital Clinical Laboratory on St. Croix with a Kodak Ektachem 700 Analyzer (Eastman Kodak Company, Rochester, NY) using a glucose oxidase colorimetric method. Insulin was measured using an RIA procedure (Linco Research Inc.). Cross-reactivity with proinsulin was under $0.02 \%$. Glucose and insulin values were used to estimate insulin resistance by the homeostasis model assessment (HOMA-IR) using the formula [fasting glucose $(\mathrm{mmol} / \mathrm{L}) \times$ fasting insulin $(\mu \mathrm{U} / \mathrm{mL})] / 22.5$ [24].

Data on lifestyle and psychosocial variables were collected with questionnaires at the study clinic. Smoking status and alcohol consumption were measured with "yes" or "no" responses to being a current smoker or having consumed at least one alcoholic beverage during the past month, respectively. Spirituality was measured using a 15-item subscale of Rogers' Life Attitude Inventory (LAI) [25]. Rogers conceptualizes spirituality or integrative spiritual orientation as feelings and expressions of hope, a sense of daily contentment or peace and feelings of connection to others, and a higher power [25]. The Rogers' LAI spirituality measure was selected for inclusion in the USVI study because it appeared to approximate the World Health Organization definition of spirituality as outlined in the Thirty-Seventh World Health Assembly resolution EB73.r3 [26]. Data on the reliability and validity of the LAI have been published [25]. Cronbach's alpha for this spirituality measure was 0.72 in the current sample. The USVI study also used ten additional items on the LAI to estimate psychosocial stress among participants. These ten LAI items assessed the respondent's sense of despair and feelings of disconnection from their community. This measure was thought to be appropriate for estimating the psychosocial stress the Afro-Caribbean immigrants experience in trying to assimilate into the USVI community. In the current sample, the psychosocial stress measure had a Cronbach's alpha of 0.70 .

\section{Statistical Analyses}

Statistical analyses were performed using Statistical Analysis System software version 9.2 [27]. Spearman correlation analyses were used to determine if education and other demographic factors were correlates of spirituality among the AfroCaribbean participants. To examine how the level of spirituality might influence the relationship of psychosocial stress and other study variables to the metabolic risk factors (waist circumference and insulin resistance), the spirituality variable was classified into three levels ("low," "moderate," and "high") based on the distribution of spirituality scores. Scores in the bottom third of the distribution were classified as low, those in the upper third as high, and those in the middle third as moderate. Stepwise regression analysis was then used to identify the best predictor variables for the two metabolic risk factors within each level of spirituality. Both forward and backward selection were used to arrive at the best prediction models at $\alpha=.05$. In these models, the independent variables were psychosocial stress score, gender, age, educational level, household income, years living in the USVI, current smoking, past month alcohol use, and BMI. The logarithm of the HOMA-IR score was used in the analyses because of the skewed distribution of insulin values.

\section{Results}

Among the study participants, $67.3 \%$ were female, nearly half (49.5\%) indicated that they were single and $42.9 \%$ reported having less than a high school education. The mean age of the participants was $47.6 \pm 13.3$ years, and most $(78.1 \%)$ had been living in the USVI for 15 or more years.

Table 1 shows the Spearman correlation coefficients relating spirituality and psychosocial stress to the other study variables. Spirituality was significantly and positively correlated with age and being married but not with any other study variable. Psychosocial stress was significantly and inversely correlated with education, income, and being married and was significantly and positively correlated with waist circumference and HOMA-IR score. There was no significant correlation between the spirituality and psychosocial stress variables.

In Table 2, the results of stepwise regression analyses show that there were differences in the factors that were independently related to waist circumference within each level of spirituality. Among participants with a low level of spirituality, psychosocial stress score, male gender, and BMI were the 
Table 1 Spearman correlation coefficients relating scores for spirituality and psychosocial stress to demographic variables among foreign-born Afro-Caribbean persons residing in the Virgin Islands of the United States

\begin{tabular}{|c|c|c|}
\hline Study variables & $\begin{array}{l}\text { Spirituality } \\
\text { correlation } \\
\text { coefficient }(r) \\
N=333\end{array}$ & $\begin{array}{l}\text { Psychosocial stress } \\
\text { correlation } \\
\text { coefficient }(r) \\
N=333\end{array}$ \\
\hline Age (years) & $0.161^{* *}$ & 0.011 \\
\hline Gender $($ female $=1$, male $=0)$ & 0.080 & 0.017 \\
\hline Educational level & -0.060 & $-0.168 * *$ \\
\hline Household income & -0.009 & $-0.179^{* *}$ \\
\hline $\begin{array}{l}\text { Marital status } \\
\quad(\text { married }=1, \text { other }=0)\end{array}$ & $0.117^{*}$ & $-0.168 * *$ \\
\hline Years living in USVI & 0.009 & -0.030 \\
\hline $\begin{array}{l}\text { Past month alcohol } \\
\quad(1=\text { yes, } 0=\text { no })\end{array}$ & -0.071 & 0.043 \\
\hline $\begin{array}{l}\text { Current smoker } \\
\quad(1=\text { yes, } 0=\text { no })\end{array}$ & -0.076 & 0.022 \\
\hline Psychosocial stress score & -0.005 & 1.000 \\
\hline Body mass index & 0.072 & 0.035 \\
\hline Waist circumference & 0.108 & $0.164 * *$ \\
\hline HOMA-IR score & 0.015 & $0.134 *$ \\
\hline
\end{tabular}

Values are significant: ${ }^{*} p<.05 ;{ }^{*} p<.01 ; * * * p<.001$

independent predictors of waist circumference. These three factors explained approximately $68.7 \%$ of the variance in waist circumference scores among participants with a low level of spirituality. Among persons with a moderate level of spirituality, the factors independently associated with waist circumference were male gender and BMI. Education (inversely associated) and BMI were the best predictors of waist circumference among participants with a high level of spirituality. The variables that were significant among participants with moderate and high levels of spirituality accounted for approximately 42.5 and $39.8 \%$ of the variance in waist circumference scores in each spirituality group, respectively.

The results in Table 3 show that the significant predictors of HOMA-IR among persons with a low level of spirituality were psychosocial stress (positively associated), past month alcohol consumption (inversely associated), and BMI. At moderate and high levels of spirituality, only BMI remained as an independent predictor of HOMA insulin resistance. The significant factors at low, moderate, and high levels of spirituality accounted for approximately $20.6,23.1$, and $18.2 \%$ of the variance in HOMA-IR values in each spirituality group, respectively.

\section{Discussion}

Spirituality has been shown to be an important aspect of the lives of both African American and Afro-Caribbean persons residing in the USA [28]. However, very few data have been published which relate spirituality to quantitative measures of health or disease risk in these groups. To add to the literature in this area, the current study examined how the factors significantly associated with waist circumference and insulin resistance would vary by level of spirituality among foreign-born Afro-Caribbean adults. Findings from these assessments, which show that psychosocial stress is significantly related to both waist circumference and insulin resistance only at a low level of spirituality, are consistent with the a priori hypothesis that higher levels of spirituality will have an attenuating effect on the relationship of psychosocial stress to stressrelated metabolic risk factors among Afro-Caribbean immigrants. It is also important to note that consistent with results for Afro-Caribbean immigrants living on the US mainland [8], spirituality was not associated with the indicators of socioeconomic status among the Afro-Caribbean participants in the current study. An obvious implication of this finding is that the health protective benefits of higher levels of spirituality are likely to extend across all socioeconomic strata among AfroCaribbean immigrants in the USVI and USA.

Given the lack of a significant association of spirituality with psychosocial stress in the current study, it appears that the
Table 2 Results of stepwise regression analyses showing parameter estimates with standard errors (SE) for significant predictors of waist circumference by level of spirituality among AfroCaribbean immigrants living in the US Virgin Islands, 1995-1999

Only variables that met the $\alpha=.05$ level of significance were included in the models. The spirituality levels included 109 persons with low spirituality, 121 persons with moderate spirituality, and 103 persons with high spirituality

\begin{tabular}{llll}
\hline & $\begin{array}{l}\text { Low spirituality } \\
\text { parameter (SE) }\end{array}$ & $\begin{array}{l}\text { Moderate spirituality } \\
\text { parameter (SE) }\end{array}$ & $\begin{array}{l}\text { High spirituality } \\
\text { parameter (SE) }\end{array}$ \\
\hline $\begin{array}{l}\text { Age (years) } \\
\text { Gender }(0=\text { male, } 1=\text { female) }\end{array}$ & $-8.260(1.548)$ & $-10.079(2.954)$ & \\
$\begin{array}{l}\text { Education (grade level) } \\
\text { Income (dollars) }\end{array}$ & & & $-1.861(0.28)$ \\
Smoking $(0=$ no, $1=$ yes $)$ & & & \\
Alcohol past month $(0=$ no, $1=$ yes) & & & \\
Time living in USVI (years) & & & \\
Psychosocial distress score & $9.080(2.438)$ & & $0.909(0.155)$ \\
BMI $\left(\mathrm{kg} / \mathrm{m}^{2}\right)$ & $1.769(0.150)$ & $2.401(0.285)$ & 0.398 \\
Model $R^{2}$ & 0.687 & 0.425 & \\
\hline
\end{tabular}


Table 3 Results of stepwise regression analyses showing parameter estimates with standard errors (SE) for significant predictors of HOMA-IR by level of spirituality among Afro-Caribbean immigrants living in the US Virgin Islands, 1995-1999

Low spirituality parameter (SE)

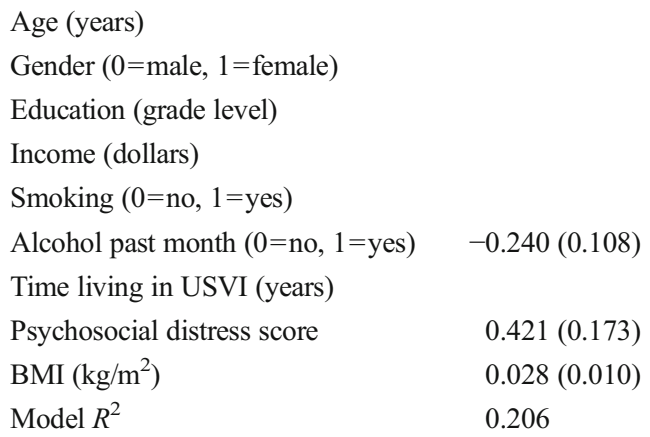

$0.051(0.009)$

0.231
$0.034(0.008)$

0.182

Only variables that met the $\alpha=.05$ level of significance were included in the models. The spirituality levels included 109 persons with low spirituality, 121 persons with moderate spirituality, and 103 persons with high spirituality

attenuating effect of spirituality on the relationship between psychosocial stress and the metabolic risk factors is indirect and results from some intermediary factor which is positively associated with spirituality and also buffers the physiological effects of psychosocial stress. Prior research studies indicate that a positive relationship exists between spirituality and coping strategies that are associated with reduced levels of emotional stress and the stress hormone cortisol [11, 29]. There is also convincing evidence that the chronic activation of the hypothalamic-pituitary-adrenal (HPA) axis by unresolved emotional stress is linked to irregularities in cortisol homeostasis and consequent abdominal obesity and insulin resistance $[12,13,30]$. Therefore, an argument can be made that the attenuating effect of spirituality on the association of psychosocial stress to waist circumference and HOMA insulin resistance in the current study might be due to a greater use of positive coping strategies among study participants who have higher levels of spirituality. However, an alternative but related explanation for the failure to find a significant association of psychosocial stress with the metabolic factors at higher levels of spirituality is that rather than promote an increase in the use of positive coping strategies, per se, higher levels of spirituality might somehow reduce the reliance on poor coping strategies which act as barriers to the resolution of emotional distress. This viewpoint is supported by results from a study of Afro-Caribbean women on the Caribbean island of Dominica which showed that poor cortisol homeostasis and higher levels of perceived stress were associated with the use of certain types of poor coping strategies but were not associated with the use of any type of positive coping strategy [31]. Further research among Afro-Caribbean populations is needed to create a better understanding of the role that spirituality plays in influencing coping choices that are linked to cortisol dysregulation and its consequent metabolic abnormalities in this ethnic group. Currently, the spirituality-coping-stress- neuroendocrine relationships are not well understood but might be important avenues for future studies to identify reasons for race and ethnic health disparities in chronic disease risk and outcomes.

In the current study, past month alcohol use was inversely associated with HOMA insulin resistance only among persons with a low level of spirituality. The inverse association of past month alcohol use with HOMA insulin resistance is consistent with results of prior research which show a positive effect of alcohol consumption on insulin sensitivity [32]. However, in contrast to results from studies among African Americans [33, 34], there was no significant correlation between spirituality and past month alcohol use among participants in the current study. One explanation for these conflicting results is that past month alcohol use may be too imprecise for a measure to detect a direct association of alcohol consumption with spirituality in the study population. Therefore, it is possible that the significant relationship of past month alcohol use with HOMA insulin resistance among participants with a low level of spirituality might reflect their consumption, on average, of a larger quantity of alcohol compared to participants with higher levels of spirituality. Also, given that alcohol consumption is often used as a method of coping with stress [35], the apparent attenuating effect of higher levels of spirituality may be due to an increase in the use of other coping strategies and less of a reliance on alcohol as a means of coping.

Overall, the factors found to be significantly related to both waist circumference and HOMA insulin resistance in the study varied by level of spirituality. Of the prediction models generated within each level of spirituality, the best overall model accounted for approximately $68 \%$ of the variance in waist circumference measurement among persons with a low level of spirituality. Among persons with moderate and high levels of spirituality, the significant predictive variables explained less than $50 \%$ of the variance in waist circumference 
and insulin resistance. These findings indicate that there are other risk correlates in addition to the ones assessed in the current study that account for a significant proportion of the variance in waist circumference and HOMA-IR scores among the Afro-Caribbean immigrants living in the USVI. It is possible that these factors might also vary by level of spirituality. Additional research is needed to elucidate the mechanistic pathways through which spirituality influences health protective behaviors and the extent to which these pathways influence race, ethnic, and nativity differences in chronic disease risk factors and outcomes.

There are some strengths and limitations to the current study that should be noted. The strength of this investigation is that the data were collected from a population-based random sample with a robust participation rate which allows for the generalization of the study results to the wider population of Afro-Caribbean immigrants living in the USVI. Data presented in the current report are cross-sectional; therefore, causal relationships cannot be implied from the significant associations that were reported. Another limitation is that alcohol use was estimated from a single variable that assessed past month use which is imprecise. It is possible that the actual quantity of alcohol consumed varies significantly among persons who report consumption in the past month.

\section{Conclusion}

In discussing the history of the USVI in the mid to late twentieth century, Dookhan [18] notes that soon after their arrival to the territory, Afro-Caribbean immigrants were resented by the local residents with whom they were competing for jobs, subjected to discrimination because they were immigrants, and were stigmatized as "aliens," a term used by the US Immigration and Naturalization Service to describe foreigners living in US territory. In this sociocultural milieu, a sense of being an outsider may have contributed to the psychosocial stress that was assessed with the measure of feelings of despair and disconnection utilized in the current study. Also, consistent with the notion that the Afro-Caribbean immigrants in the USVI were differentially impacted by this type of stress, a previous study in the USVI found that psychosocial stress as measured by feelings of despair and disconnection was associated with waist circumference and insulin resistance among Afro-Caribbean immigrants but not nativeborn Afro-Caribbean persons [21]. It is not unreasonable to hypothesize that, among Afro-Caribbeans and African Americans in the USA, experiences of discrimination and racism [36] might also contribute stress associated with feelings of being an outsider in the community in which they reside or in the country as a whole. Therefore, the finding that the association of this type of psychosocial stress with the metabolic risk factors was attenuated by spirituality in the current study has intriguing policy and programmatic implications for efforts to address chronic disease risk factors like abdominal obesity and insulin resistance among black Americans in the USVI and USA.

In a general sense, the results of the current study suggest that efforts by policy makers and civic leaders to foster social cohesion and feelings of inclusion among all ethnic and nativity groups within the USVI community should be seen as important for lessening the effect of psychosocial stress on chronic disease risk in the population. Such efforts may be more challenging in the larger USA but are equally important. More specifically, Afro-Caribbean immigrants in the USVI might benefit from the incorporation of spirituality concepts such as sense of hope, contentment, and connectedness into clinical and public health prevention programs targeted at reducing type 2 diabetes and cardiovascular disease risk. As suggested by the current study, this strategy might not only serve to lessen the influence of psychosocial stress on the related metabolic risk factors among Afro-Caribbean immigrants but might also foster improved practice of other health protective behaviors. Specific strategies for doing this can be adopted from existing programs in the USA that have been successful in incorporating spirituality concepts into clinical interventions to mitigate low birth weight [37], which like abdominal obesity is associated with stress-induced cortisol dysregulation $[30,38]$.

Acknowledgments This study was supported by a grant (RO1 DK46502) from the National Institute for Diabetes, Digestive and Kidney Diseases, National Institutes of Health, USA.

Conflict of Interest The authors, Eugene S. Tull, Willa M. Doswell, and Malcolm A. Cort, declare that they have no conflicts of interest.

Informed Consent All procedures followed were in accordance with the ethical standards of the responsible committee on human experimentation (institutional and national) and with the Helsinki Declaration of 1975 , as revised in 2000. Informed consent was obtained from all patients for being included in the study.

\section{References}

1. Nickens H. Report of the secretary's task force on black and minority health: a summary and presentation of health data with regards to blacks. J Natl Med Assoc. 1986;78:577-80.

2. Golden SH, Brown A, Cauley JA, Chin MH, Gary-Webb TL, Kim C, et al. Health disparities in endocrine disorders: biological, clinical and non-clinical factors - an endocrine society scientific statement. J Clin Endocrinol Metab. 2012;97:E1597-639.

3. National Institute of Medicine (US) Committee on Understanding and Eliminating Racial and Ethnic Disparities in Health Care. Unequal treatment: confronting racial and ethnic disparities in health care. Smedley BD, Stith AY, Nelson AR, editors. Washington (DC): National Academies; 2003

4. Singh GK, Hiatt RA. Trends and disparities in socioeconomic and behavioural characteristics, life expectancy, and cause-specific 
mortality of native-born and foreign-born populations in the United States, 1979-2003. Int J Epidemiol. 2006;35:903-19.

5. Bediako SM, Griffith DM. Eliminating racial/ ethnic health disparities: reconsidering comparative approaches. J Health Dispar Res Pract. 2007;2:49-62.

6. Griffith DM, Moy E, Reischl TM, Dayton E. National data for monitoring and evaluating racial and ethnic health inequities: where do we go from here? Health Educ Behav. 2006;33:470-87.

7. Griffith DM, Johnson JL, Zhang R, Neighbors HW, Jackson JS. Ethnicity, nativity, and the health of American blacks. J Health Care Pore Underserved. 2011;22:142-56.

8. Taylor RJ, Chatters LM, Jackson JS. Correlates of spirituality among African Americans and Caribbean blacks in the United States: findings from the national survey of American life. J Black Psychol. 2009;1(35):317-42.

9. Lee EK, Chan K. Religious/spiritual and other adaptive coping strategies among Chinese American older immigrants. J Gerontol Soc Work. 2009;52:517-33.

10. Lee KH, Yoon DP. Factors influencing the general well-being of lowincome Korean immigrant elders. Soc Work. 2011;56:269-79.

11. Ironson G, Solomon GF, Balbin EG, O'Cleirigh C, George A, Kumar $\mathrm{M}$, et al. The Ironson-woods spirituality/religiousness index is associated with long survival, health behaviors, less distress, and low cortisol in people with HIV/AIDS. Ann Behav Med. 2002;24:34-48.

12. Bjorntorp P. Visceral fat accumulation: the missing link between psychosocial factors and cardiovascular disease? J Intern Med. 1991;230:195-201.

13. Roseman R, Dallman MF, Bjorntorp P. Stress-related cortisol secretion in men, relationships with abdominal obesity and endocrine metabolic and hemodynamic abnormalities. J Clin Endocrinol Metab. 1998;83:1853-9.

14. Meigs JB, Rutter MK, Sullivan LM, Fox CS, D'Agostino Sr RB, Wilson PW. Impact of insulin resistance on risk of type 2 diabetes and cardiovascular disease in people with metabolic syndrome. Diabetes Care. 2007;30:1219-25.

15. Krieger N, Kosheleva A, Waterman PD, Chen JT, Beckfield J, Kiang MV. 50-year trends in US socioeconomic inequalities in health: USborn black and white Americans, 1959-2008. Int J Epidemiol. 2014 Mar 16. [Epub ahead of print]

16. Goodman E1, Daniels SR, Dolan LM. Socioeconomic disparities in insulin resistance: results from the Princeton school district study. Psychosom Med. 2007;69:61-7.

17. US Department of State. Purchase of the United States Virgin Islands, 1917. US. Department of State website. http://2001-2009.state.gov/r/ pa/ho/time/wwi/107293.htm. Accessed August 8, 2014

18. Dookhan I. A history of the Virgin Islands of the United States. Mona, Jamaica: Canoe Press, University of the West Indies; 1994.

19. Lewin A. Census shows V.I.'s population down 2\%. The Virgin Islands Daily News. August 25, 2011. http://virginislandsdailynews. com/news/census-shows-v-i-s-population-down-2-1.1193325. Accessed June 5, 2014

20. Tull ES, LaPorte R, Kriska A, Mark J, Hatcher AT. Glucose intolerance by race and ethnicity in the U.S. Virgin Islands. J Natl Med Assoc. 2002;94:135-42.

21. Tull ES, Thurland A, LaPorte RE. Acculturation and psychosocial stress show differential relationships to insulin resistance and body size in two groups of blacks living in the U.S. Virgin Islands. J Natl Med Assoc. 2003;95:650-575.
22. The Arlie (VA) Consensus Conference. In: Loham T., Roche A, Martorel R. editors. Standardization of anthropometric measurements. Champaign, IL: Human Kinetics, 1988. pp: 39-80

23. Barrow JS, Webster J. Quetelet's index (W/H2) as a measure of fatness. Intl J Obes. 1985;9:147-53.

24. Matthews DR, Hosker JP, Rudenski AS, Naylor BA, Treacher DF, Turner RC. Homeostasis model assessment: insulin resistance and beta-cell function from fasting plasma glucose and insulin concentrations in man. Diabetologia. 1985;28:412-9.

25. Jackson-Lowman H, Rogers JA, Zhang X, Zhao Y, Brathwaite-Tull M. Life attitude inventory: preliminary evaluation of a measure of spiritual orientation. In: Jones RL, editor. Handbook of tests and measurements for black populations. Hampton: Cobb \& Henry; 1996. p. 87-103.

26. World Health Organization (1984). Global strategy for health for all by the year 2000: the spiritual dimension. Report of the DirectorGeneral to facilitate discussion of the Executive Board, EB73/1984/ REC/I, Annex 1, page 23

27. SAS Institute Inc. SAS /STAT 9.2 User's Guide, Cary, NC: SAS Institute Inc., 2008.

28. Linda M, Chatters LM, Robert J, Taylor RJ, Bullard KM, Jackson JS. Spirituality and subjective religiosity among African Americans, Caribbean blacks and Non-Hispanic whites. J Sci Study Relig. 2008:47:725-37.

29. Carrico AW, Ironson G, Antoni MH, Lechner SC, Durán RE, Kumar $\mathrm{M}$, et al. A path model of the effects of spirituality on depressive symptoms and 24-h urinary-free cortisol in HIV-positive persons. J Psychosom Res. 2006;61:51-8.

30. Rosmond R, Dallman M, Bjorntop P. Stress-related cortisol secretion in men: relationships with abdominal obesity and endocrine, metabolic and hemodynamic abnormalities. J Clin Endocrinol Metab. 1998;83:1853-9.

31. Tull ES, Sheu YT, Butler C, Cornelius K. Relationships between perceived stress, coping behavior, and cortisol secretion in African Caribbean women with high and low levels of internalized racism. J Natl Med Assn. 2005;97:206-12.

32. Bell RA1, Mayer-Davis EJ, Martin MA, D'Agostino Jr RB, Haffner SM. Associations between alcohol consumption and insulin sensitivity and cardiovascular disease risk factors: the insulin resistance and Atherosclerosis study. Diabetes Care. 2000;23:1630-6.

33. Reeves RR, Adams CE, Dubbert PM, Hickson DA, Wyatt SB. Are religiosity and spirituality associated with obesity among African Americans in the Southeastern United States (the Jackson Heart Study)? J Relig Health. 2012;51:32-48.

34. Staton-Tindall M, Duvall J, Stevens-Watkins D, Oser CB. The roles of spirituality in the relationship between traumatic life events, mental health, and drug use among African American women from one southern state. Subst Use Misuse. 2013;48:1246-57.

35. Grant VV, Stewart SH, Mohr CD. Coping-anxiety and copingdepression motives predict different daily mood-drinking relationships. Psychol Addict Behav. 2009;23:226-37.

36. Krieger N, Kosheleva A, Waterman PD, Chen JT, Koenen K. Racial discrimination, psychological distress, and self-rated health among US-born and foreign-born black Americans. Am J Public Health. 2011;101:1704-13.

37. Breen GV, Price S, Lake M. Spirituality and high-risk pregnancy: another aspect of patient care. AWHONN Lifelines. 2006;10:466-73.

38. Duthie L, Reynolds RM. Changes in the maternal hypothalamicpituitary-adrenal axis in pregnancy and postpartum: influences on maternal and fetal outcomes. Neuroendocrinology. 2013;98:106-15. 